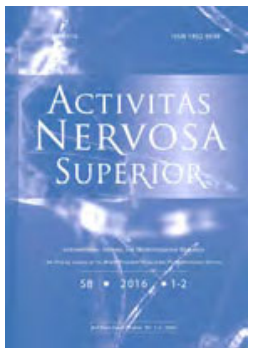

\title{
NON-TRADITIONAL INTERPRETATION OF CONVENTIONAL EEG CURVE ANALYSES
}

\author{
Josef Faber 1,2*, Mirko Novak², Zdenek Votruba², Michal Kovaljov² \\ ${ }^{1}$ Department of Psychiatry, First Faculty of Medicine, Charles University, Prague, Czech Republic \\ ${ }^{2}$ Czech Technical University, Faculty of Transportation, Prague, Czech Republic
}

\begin{abstract}
The electroencephalographic (EEG) curve is a highly complex formation representing cerebral system activities. On the one hand it may resemble featureless oscillations in alpha activity, on the other hand it looks like a very irregular tangle of mostly quite random lines even when the proband or patient is in a waking state. Recent findings also indicate that the EEG signal can be studied as a complex numerical series using signal analysis in terms of chaodynamic processes and describe its outcome as fractals or attractors. Using spectral Gabor analysis (GA), local coherence (LCA) and amplitude analyses (AA) we report results with regard to clinical experience and atractor character of epileptic activity. We have developed a simple coefficient method showing the state of EEG synchronisation and, at the same time, also the actual state of integration or complexity of the systems in the brain. Amplitude analysis shows the fractal feature of alpha activity and attractor descriptions related to of epileptic activity. These results might be useful for detection of mental states related to levels of wakefulness or somnolence.
\end{abstract}

Key words: EEG analysis; Atractor; Fractal; Chaodynamic system

\section{INTRODUCTION}

While reflexes are the basic elements of nervous activity, their mechanisms are of diverse structural complexity, hence, with regard to their complexity, we classify them in different hierarchical degrees. The activity of spinal cord takes the form of variol reflexes for example myotatic reflexes, Babinski reflex, stepwise mechanism and reflexes related to sneezing, breathing, vomiting, coughing, (Vitek 1951, Rimbaud 1957, Ambler et al. 2004, Petrovicky et al. 2008). In addition these functions are linked to the limbic system and hypothalamus activities which is related to rudimentary motivation, impulses, incentives, Ukhtomski dominants etc. (Atwood \& MacKay, 1989; Atkinson et al., 1995; Faber, 2005).

The hierarchy of mentation is is present also in subhuman animals (Cragg, 1975) in the form of abstract mentation related to a rich genetic (instinctive) library containing information for sensory analysis and for motor reactions (North \& Greenspan 2007).

Reflexological development continues to reach more complex conditioned reflexes and their sequences in the form of dynamic stereotypes that can be assessed using EEG for example cognitive evoked potentials for example P300, N400, N600. As Kukleta et al. (2009)

${ }^{*}$ Correspondence to: Josef Faber, Department of. Psychiatry, Ke Karlovu 11, 12000 Prague 2, Czech Republic Received January 28, 2016; accepted February 22, 2016; Act Nerv Super 58(1-2), 28-44; ISSN-1802-9698 
show both their extensive localisation from the frontal to the parietal area and their association with movement. Primitive mentation and movement are no doubt closely related. In their development, motor mentation is followed by imaginative mentation with abstract propositional mentation which occupies the peak position. The first two types are obviously functional in children and in paradoxical sleep dreams, i.e. they are independent of age.

It is possible that biological neuronal structures (nets $=\mathrm{BNN}$ ) of the spinal cord and brain stem are determined mainly genetically, preformedly, that is by nature, i.e. deductively. In other words, they are controlled by adnate programs repeatedly put to use and contained in "well-tried genes". From the neuroinformative (neurocybernetic) point of view, they appear to be morphologically petrified attractors (see further on). The cortex (with its BNN) is constructed in a similar way, also genetically, except that its postnatal development is more inductive than deductive, not preformed, but developing under the influence of "nurture". The trajectories of the cortex as a system are more of the type of fractals (Faber 2005, 2015). Michel Jouvet, an outstanding French neurophysiologist and sleep resercher, describes paradoxical sleep as genetic programming of the brain: motor patterns acquired during the day are, by way of cooperation with DNA, transcription, translation, splicing and proteinogenesis petrified into proteins during nocturnal sleep.

In postnatal life learning is indispensible. According to that well-known Canadian neurosurgeon W. Penfield, learning is brain programming. Learning is reception of new information from the environment followed by logical organization of biological nervous networks (BNN) in the thalamocortical reverberation system (TCRS). In the limbic system learning is under the effect of the individuality of the personage (subjective emotional filter) and genetic mechanisms of memory. Maturation is a term used to describe spontaneous development of some "skill" (nature), e.g. the swallow goes without having to learn how to fly like, say, the eagle, instead, it leaves its nest at first attempt as a fairly good flier.Vultures have inborn use of a stone for breaking other's eggs for eating. Deprivation follow-up shows that while it takes a young vulture long months to learn the skill, it will learn it all the same. Although this "instinctive learning" takes so long, that skill is more of an inborn gift. Hence, it belongs rather in the area of "nature", i.e. development of behaviour resembling (geometrically speaking) a point or cyclic trajectory of an attractor in the complex system of the brain (see further on).

Neurohistologists estimate the number of neurons in the cortex differently, the number ranges between 15 up to 100 billion nerve cells with the number of synapses going to billions and trillions. When estimating psychic properties of the brain such as cognition or concrete or abstract mentation such numerical differences are negligible since in the end none of us can imagine a meaningful coordination of dozens of billions of active elements within a single system. At the same time, it is hard to expect such a number of neurons to operate according to deterministic algorithms.

Whether or not we want it in a system so large and so intricately interconnected, we must expect random data and unpredictable processes, i.e. noise. Random impulses develop as early as months $3-4$ of the foetal brain as primarily generated impulses and remain there in the random regimen for the rest of the subject' life. Cragg (1975) presents facts about prenatal foetuses of cats, whose neurons, synapses and glia, the maturity of which is far from accomplished, while their intercellular connections are already partially founded. This means that hardware runs ahead of software, though the effect of genetics (nature) and sensory information (nurture) are already well balanced after birth (Trojan \& Stastny, 1980).

For example, Valverde (1971) found exponential increase in the number of dendritic spines of pyramidal neurons of the murine optic cortex from birth up to the 20th postnatal day, whereas mice kept in the dark had their cell spines and thereby also synapses considerably underdeveloped. In some of the animals transferred on day 20 from the dark to light, nerve spines retardation was only partially reversible.

In the postnatal period, meaningful signals have an increasingly important role to play in the development of membrane receptors, dendritic spines and synapses in BNN. In this 
respect it seems strange that interhuman verbal communication can exist at such a high level of random interneuronal communication. Here, however, biological cultural development gave rise to coding in the form of national languages or supranational special means of communication such as the hallmarks and symbols of music (notes), mathematics, chemistry, physics or logic under the pressure of the need for communication in society. Indeed, under the pressure of external environment, even wild animals developed a nonverbal "language" of gestures, mimics, feromons and related rules of nutritional, sexual or territorial behaviour.

Yet even so, the human society is troubled with a great deal of misunderstanding arising from "dyscommunication" with serious consequences such as "neighbourly" discords as well as conflicts taking the form of "cold" or "hot" wars. Despite all the progress, it is still impossible to decide about the share of meaningful as distinct from random signals in the brain (Lion \& Winter,1953; Saunders,1963; Faber, 1978; Faber \& Vladyka, 1984). According to Bob (2005) , interaction between noise and information is not simple to define even at the level of "conscious" functions; for example, a sixth of psychotics and a third of epileptics suffer from dissociated states, i.e. to a certain degree of disintegration of psychic functions involved in consciousness, and their shift to mistakenness and randomness. Kukleta et al. (2009) point to the correlation between cognitive and motor functions, which is discernible in medial electrodes situated in frontal up to parietal areas (in vertex).

It is also possible that the motor system as such has a profound effect on the organization of noise, cognition and mentation. Many authors also suggest the need for correct cooperation of a number areas during the perception of complex pictures such as the human face and eye movements (areas 19 and 8) or speech function and perception areas $(40,41,21,22)$. Each Brodmann area contains cortical layers 3 and 5 of pyramidal ("motor") cells for communication with the same layers of other areas and also with subcortical structures.

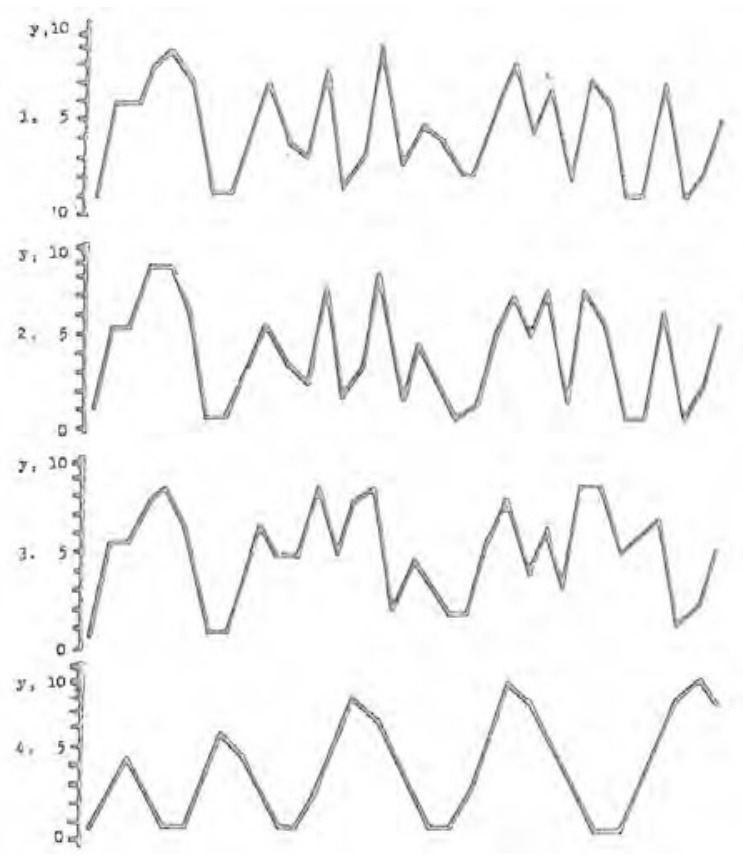

Figure 1. Four curves with various quantity of both regular and random variables.

Just for illustration of above described theoretical interpretations of recent well established findings, the Figure 1 shows 4 curves with various quantity of both regular and random variables. Using analogo-digital conversions the curves were converted to a numerical series 
for analysis with Wilks' periodogram which permits to separate harmonic (regular) from random (noise) variables. Curve No. 1 from above contains solely random variables ranging from 1 to 9 (on axis $y$ ) and was constructed using random number table. The statistical distribution is well balanced, i.e., all the numbers have the same probabiliy of incidence in this set of one-figure numbers: 1,5,5,8,9,7,1,1,4,7,4,4, etc. The total number of numbers in each set is $n=500$. The following curve 2 resulted from curve 1 by adding a regular periodic variable: number 1 to every number in the order of 4 and 5, and again number 1 was substracted from every number in the order of 9 and 10. Consequently, curve 2 contains $4,4 \%$ of regular variables. Curve 3 was enriched similarly but in double the amount. The fourth curve is the model of the first half of the alpha spindle, now entirely without any random variables.

The results of analysis of the above curves obtained with Wilks periodogram: multiple correlation coefficient (MCC) shows in curve 1 nonsignificant harmonic variables $(\mathrm{MCC}=$ 0.13 ) showing that "all" that is present is noise. However the following two curves ( 2 and 3 ) already have statistically significant variables with MCC showing 0.20 and 0.37 resp. MCC for alpha activity is 0.97 , but then, this is an entirely determined artificial curve.

Consequently, even an intensively noise affected curve carrying a mere $4.4 \%$ of harmonic components is able to carry meaningful information. This analysis is able to detect a minimum of information in a set containing $95.6 \%$ (!) of noise

Presumably enough, a similar signal-to-noise situation may exist also in brain structures $(\mathrm{BNN})$ and contain similar and certainly even more advanced filters than our artificial mechanism in ANN such as FFT, Gabor's spectral filtration, signal prediction by means of ANN, principal component method (analysis), higher order polynomials, etc. We applied Wilk's periodogram also to EEG curves of 20 patients, who, during an hour lasting EEG recording went through three states: vigilance with alpha waves, NONREM 2 sleep (with theta to delta waves) and an epileptic paroxysm of absences and pseudoabsences with spikes and waves. The highest level of noise (the lowest MCC) proved to be in wakefulness, the lowest during NONREM 2 (the highest MCC) and during absence. Surprisingly enough, we noted a high predictability of the sleep curve and, on the other hand, a high level of noise during vigilance (Faber, 1978; Faber \& Vladyka, 1984).

\section{METHODS}

EEG examinations were performed in 44 persons (19 young healthy subjects aged 21 to 35 years of age, 5 patients suffering from epileptic absence paroxysms aged 42 to 51 years, and 20 children, 6 to 15 years old diagnosed with ADHD (Attention Deficit/ Hyperactivity Disorder). However, tens of such patients had been nonsystematically examined with these methods for a number of years before). Here we present and compare the results of EEG analysis in the above three groups. For this analysis we used the Gabor spectral method (GA), local coherence function (LCF) and amplitude isopotential analysis with $17 \mathrm{msec}$ intervals of isopotential lines (AA). The resulting data were interpreted, mainly in AA, somewhat non traditionally in terms of the chaodynamic theory because we expected low predictability both in EEG and in psychological reactions, as well as a large amount of random variables in the EEG curve.

The main purpose was to identify the following four states: resting state with eyes closed, epileptic paroxysm of absence, getting off to sleep and deep sleep. We introduced a coefficient in the form of a fraction wherein the numerator is " $\mathrm{m}$ " numbers expressing the sum of all maximum values in positivities and negativities in AA maps. The number " $n$ " in the denominator invariably represents the total number of electrodes, i.e. sites on the surface from where the EEG signal is recorded, i.e. 19. In this way the coefficient is found each time in the region of the right fraction from 0 to 1 . The higher the coefficient, the more regular, simpler and more synchronized is the curve. "The coefficient of complexity - synchrony heterogeneity" (c.m/n) is then found in the interval of $0 / 19$ up to $19 / 19$, or between zero and 
one. $0 / 19$ stands for the most prominent desynchronization such as in intensive attention with eyes open. 19/19 means maximum synchronization such as during an epileptic paroxysm. In this way we should like to continue reckoning hundreds of alpha waves to obtain a wider view and devise further statistical parameters. To do so, it will be necessary for the future to develop a program.

\section{RESULTS}

Coefficient $\mathrm{m} / \mathrm{n}$ in alpha activities during vigilance is on average $4 / 19$, in the paroxysm of absence $18 / 19$. during hypnagpgia $2 / 19$, in deep sleep $7 / 19$.

In children with ADHD the $\mathrm{m} / \mathrm{n}$ coefficient during alpha waves ranges from 2/19 to 4/19, and during alpha superposed on delta waves 4/19. Moreover, delta waves in the EEG of children with ADHD show reduced regularity and periodicity. Hence, this coefficient indicates fairly well the state of synchronization, heterogeneity, graphoelements and, with some licence, also the EEG curve integrity and, at the same time, the degree of vigilance.

The likelihood of the above listed results in epileptic paroxysms reaches nearly $100 \%$, during deep sleep $80-90 \%$, and in vigilance with alpha waves the reliability is a mere $50 \%$. Noteworthily enough, even in periods of lucid vigilance with regular alpha activity there are still quite a few fairly random variables.

Of considerable interest is sleep seen in terms of those analyses. The onset of sleep, i.e, hypnagogium (in general terms NONREM 1), is marked by a drop of energy throughout the spectrum and even local coherence and a low $\mathrm{m} / \mathrm{n}$ coefficient. In contrast, deep synchronous sleep (NONREM 3) shows the very opposite, namely high energy of the spectrum, mainly in the delta band, and considerable coherence and high coefficient $\mathrm{m} / \mathrm{n}$ values. This only shows the great complexity of sleep process control, a process obviously regulated by different programs at different stages of sleep.

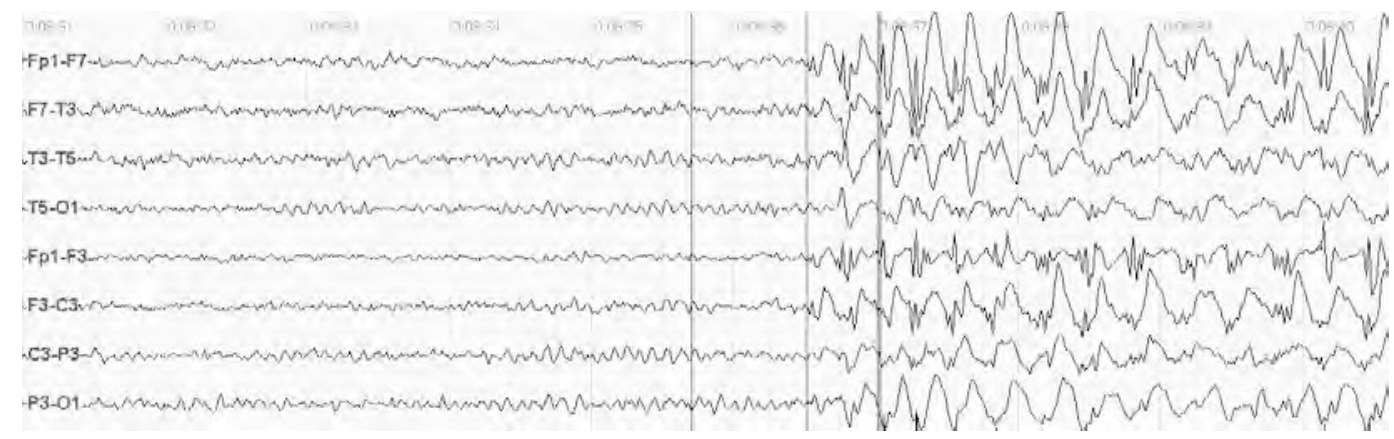

Figure 2. EEG graph of a 60-year-old waking man with irregular alpha activity in full vigilance (VIG, in the graph on the left) which, however, suddenly passes into a (semisubclinical?) epileptic (EP) paroxysm of absence with spike-wave complexes (in the graph on the right). The following two pictures map the results of alpha analysis (VIG) as well as epileptic (EP) activity.

For the sake of illustration we present EEG curves and their mathematical analysis in three subjects, representing three groups of examined probands and patients. The examples in Fig. $2,5,7,8$ are EEG records of an epileptic while waking before the attack, and during a fit of absence; in a healthy person during hypnagogia (NONREM 1) followed 25 minutes later by NONREM 3. Figures 3, 4, 6, 7, 9 show the results of analyses (GA, LCF, AA) for all four states (vigilance, absence, hypnagogia and NONREM 3). The calculation takes the form of maps in pseudocolours to facilitate orientation in the scales attached. 

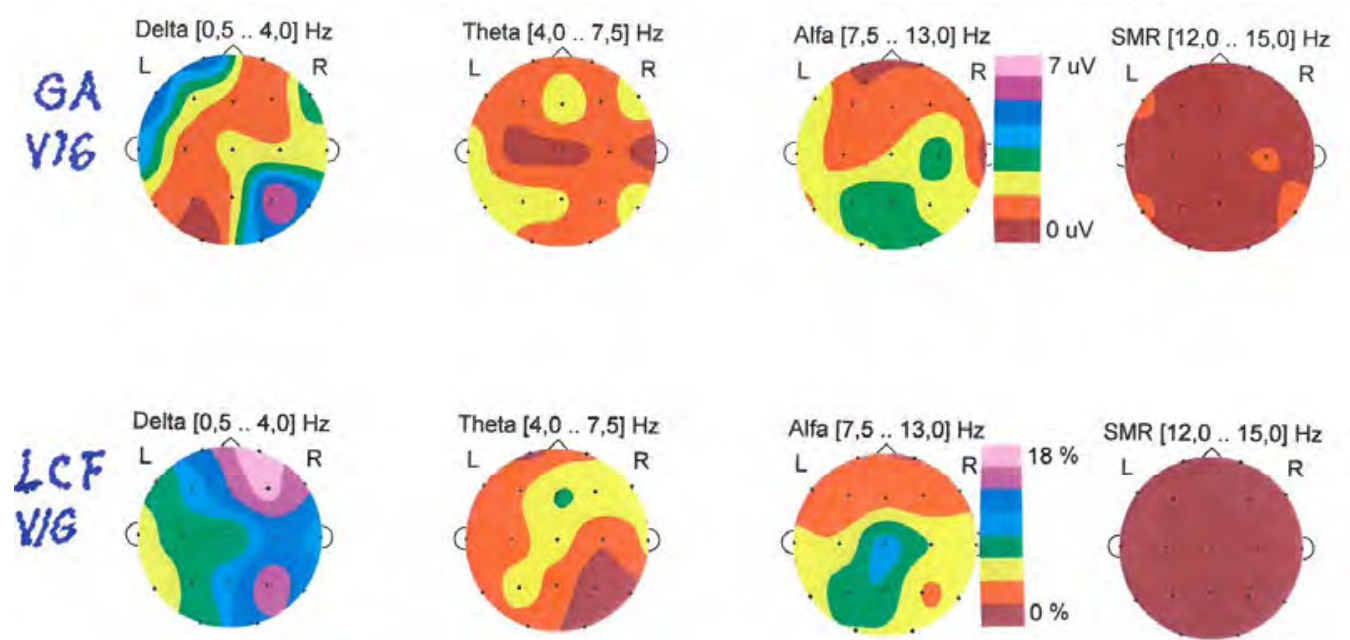

SMR $[12,0 \ldots 15,0] \mathrm{Hz}$
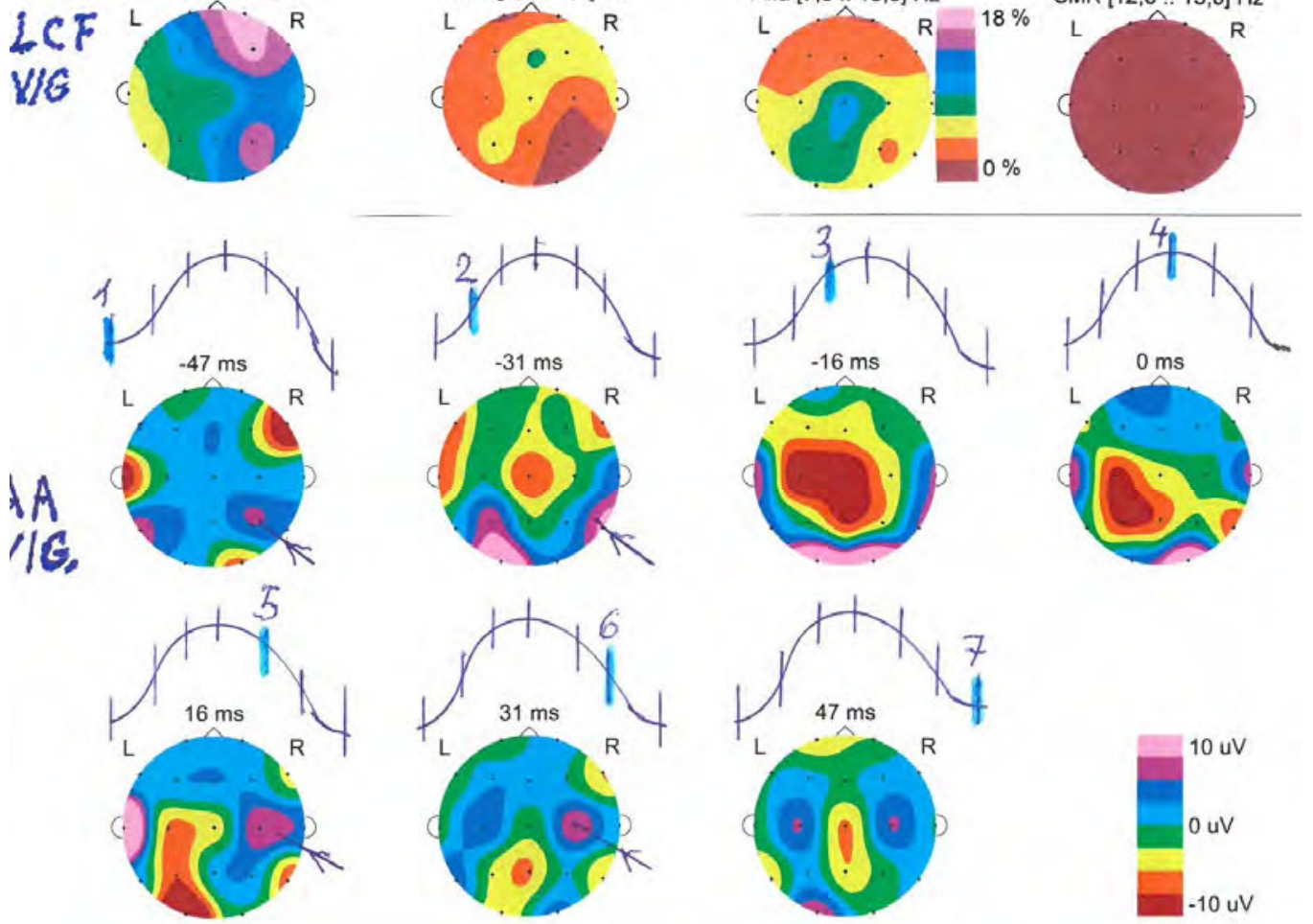

Figure 3. Upper part: Gabor analysis (GA) of alfa activity in EEG while waking (VIG) from the left side of Figure 2.

The spektrum on Figure 3 is expressed in a map for each frequency separately, in particular from the left to the right: delta, theta, alpha and slow beta. Alpha activity is almost symmetrical in typical occipital location. Delta shows focal maximum in the right temporooccipital area, possibly the site of the primary focus of the damage, or also an epileptic focus as the site of subsequently developing epileptogenesis. For quantitative orientation, colour scales are presented each time on the right in all three types of analysis (GA, LCF, AA). The middle part of the illustration shows the local coherence function (LCF) during vigilance and slightly asymmetric alpha on the left though with its maximum situated occipitally. Delta has two foci, one in the right frontal area (though, oddly enough, missing entirely from the spectrum), the other situated in the right temporoocipital area with increased coherence corresponding with the spectrum (GA). Maximum delta waves in the spectrum and in coherence are in the temporooccipital area on the right, which is repeated with a certain change also in EEG analysis of the paroxysm of absence. Beta and theta activities have no substantial role to play in the spectrum or in coherence. 
The lower part of the figure 3 shows two rows of maps with amplitude analysis (AA) of alpha activity in waking. Isovoltage maps contain data from all 19 channels. Intervals between cross sections of amplitudes have a distance of $16 \mathrm{~ms}$. Seven maps demonstrate changes of amplitude in the course of one alpha wave in a total interval of $94 \mathrm{~ms}$. To facilitate orientation, each map carries a drawing of an ideal alpha wave with a short perpendicular line indicating moments wher the "isovoltage cuts" were made.The alpha wave development is easy to see: beginning (point 1) with mild diffuse positivity (blue colour) and two small foci of major negativity parietally on the right and left. Points 2-4 show the development of major central negativity becoming smaller in points 4 and 5 . The last point (7) shows again two small foci of positivity in the central area. Apparently enough, the beginning (point 1) and the end (point 7) are like one another, consequently proving a measure of cyclicity, which, however, looks different though similar in subsequent waves. What we have here is constantly changing self likeness. Hence, the alpha rhythm has fractal characteristics, i.e. quasiperiodicity and self likeness. This might prove to be useful for a more detailed analysis of the basic alpha rhythm or also for predicting EEG curve development and thereby also an individual's behaviour. Our EEG picture consists of 19 electric amplifiers recording electric potential from 19 electrodes at different sites on the head surface, which is number " $n$ " in the fraction denominator, The number of maximum values of positivities and negativities is expressed by their sum " $\mathrm{m}$ " (here the four localities) given in the numerator of the fraction $(\mathrm{m} / \mathrm{n})$. Here, during vigilance (VIG), the coefficient c. $\mathrm{m} / \mathrm{n}$ is $4 / 19$.

The spectrum (GA) on Figure 4 again has a large delta focus with frontal right maximum, less so also theta and alpha, A smallish delta focus is situated temporoposteriorly right. Instead of slow beta (SMR- sensorimotor rhythm, here of no consequence owing to very low energy), we placed there fast beta of $20-30 \mathrm{~Hz}$ showing also a focus in the frontal right region. Instead of a delta focus frontal right, LCF in EP shows it in the right temporooccipital area. Judging from our clinical experience, EEG topic diagnosis is either not accurate, or our patients had two discernible epileptic foci. The rest of the frequences have a nearly zero coherence.

And last, AA in EP first shows maximum positivities in the centre (in maps - 47 up to 0) with a later appearance of maximum negativities ( 0 up to +47 in maps). Medium values are missing. This is proof of the presence of maximum synchronization (positive interference of rhythm genenerators), a situation to be expected in an epileptic paroxysm. Moreover, there is considerable simplification of the EEG curve which precludes any meaningful comprehensive exchange of information in the nervous network; as a result mentation is made impossible apart from loss of consciousness. Instead of complex and differentiated fractal variability, there is "pendulum" stability of a cyclic attractor in what is primitivization and dedifferentiation ("primitive superintergation“) of impulsation activity in the thalamocortical reverberation system (TCRS). Interestingly enough, though, even in this kind of maximum hypersynchronization we can discover a probable focus of epileptogenesis in the form of inclusion of negativity occasionally "making its way" there from the right temporal region, i.e., near the suspected epileptic focus. 

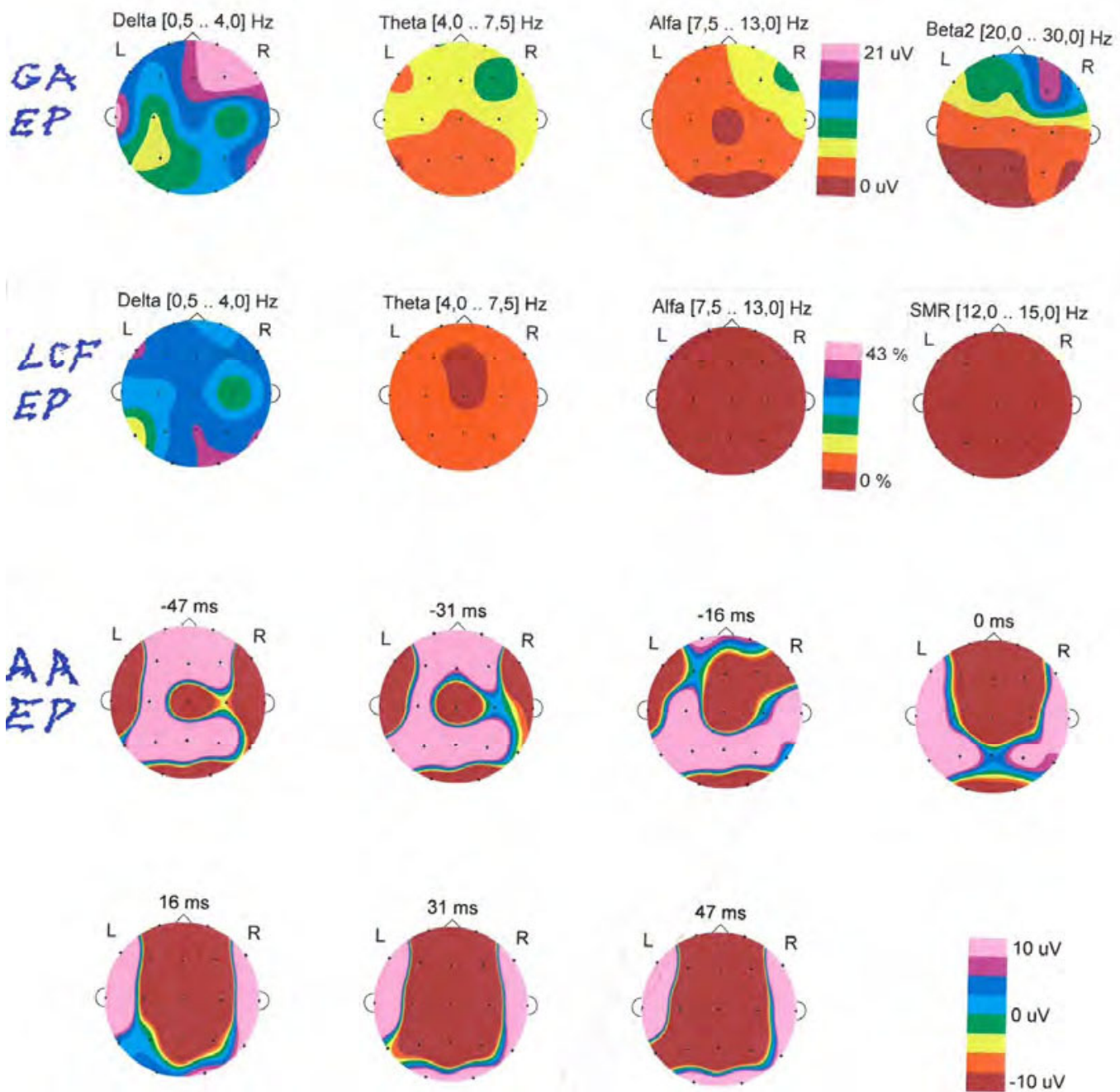

Figure 4. All three analyses were also performed in epileptic activity (EP) marked by EEG graphoelements spike-wave, right side of Fig. 2). (Technical legend for all three analyses (GA, LCF, AA) is the same as in the preceding Fig. 3).

AA analysis can again help us get closer to objective assessment of the degree of synchronization and heterogeneity, a situation favourable enough for clinical uses. Coefficient $\mathrm{m} / \mathrm{n}$ is calculated in the same way as shown in Fig.3, here in EP absence it is equal to $\mathbf{c . m} / \mathbf{n}$ 18/19, which indicates extreme synchronization, most likely complete with loss of vigilance. For that reason, rather than subclinical the seizure will be a clinical one. 


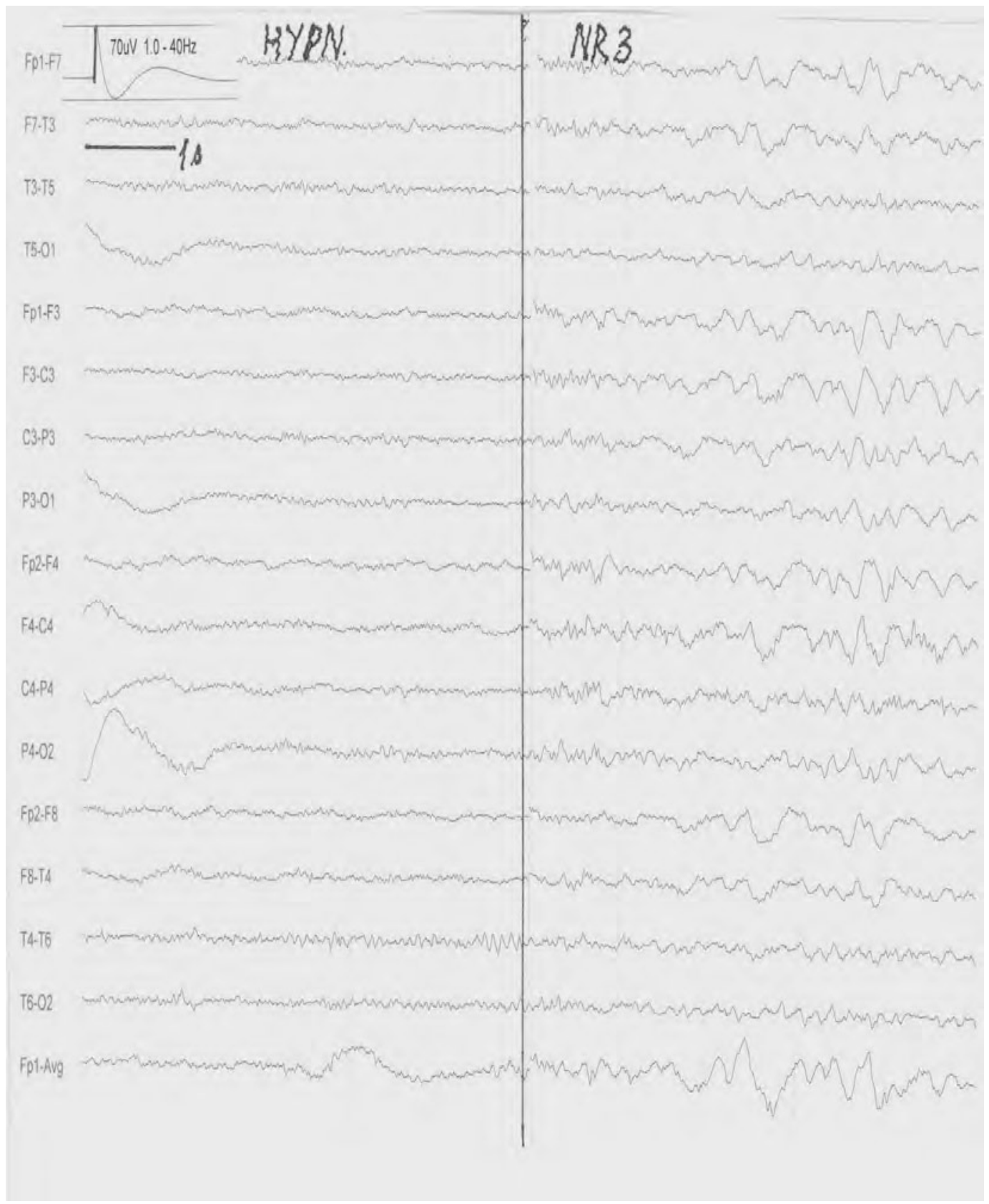

Figure 5. The left side of the illustration represents a flat curve during hypnagogia (NONREM 1, HYPN, hypnagogia), the right side deep sleep (NONREM 3) with high, on the whole, symmetrical delta and theta waves. A healthy experimental 29 -year-old person.

The following two figures will demonstrate the results of analyses of the two states - getting off to sleep and NONREM 3. 

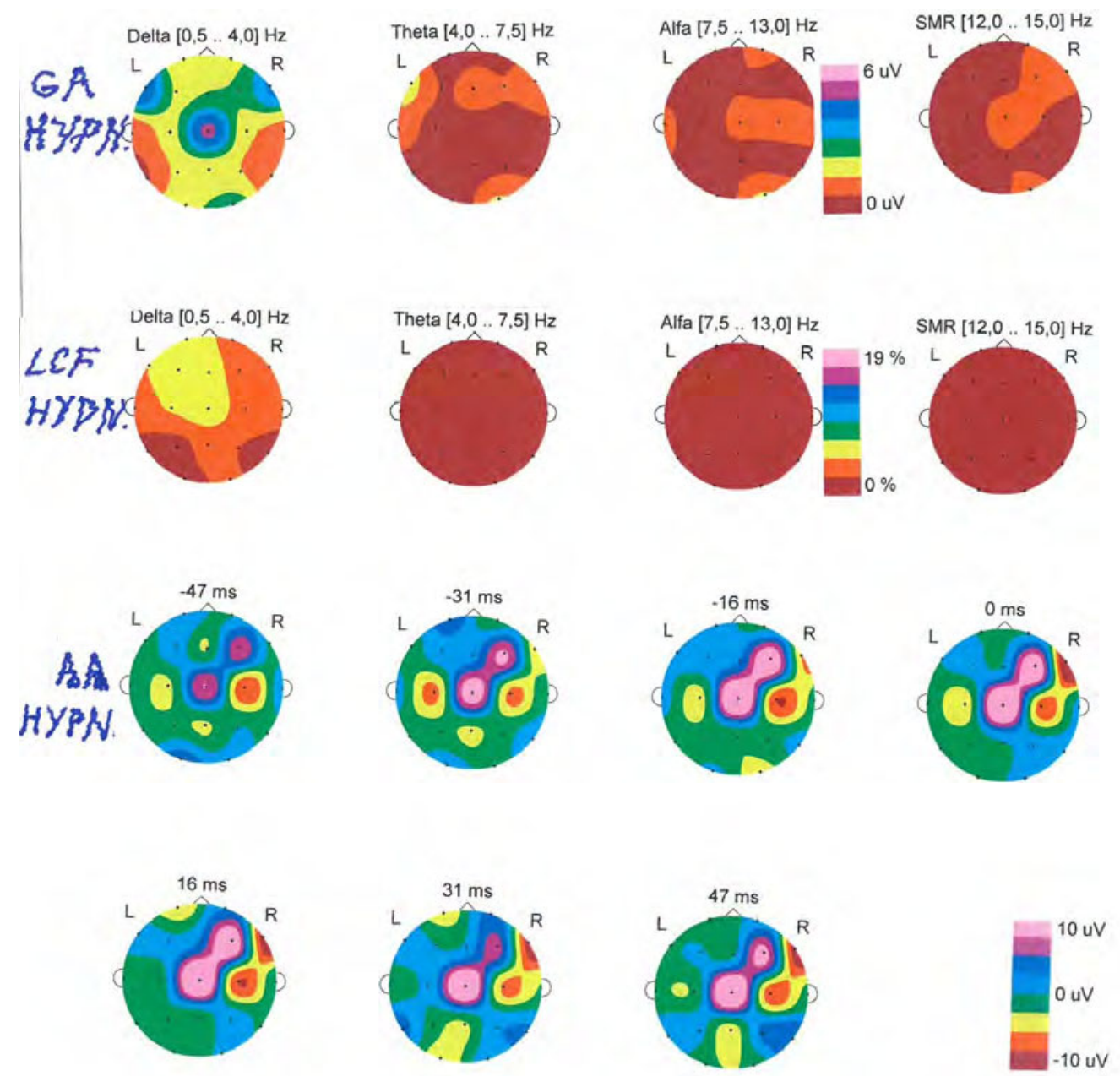

Figure 6. Analysis of the left side of Fig. 5 shows, during hypnagogia, in the spectrum (GA) central focal predominance of delta in what is generally low energy. LCF during hypnagogia has slightly increased delta of low coherence on the left. The other frequences are practically nill coherence-wise. This is evidence of a marked drop in synchronization, i.e. onset of desynchronization (negative wave interference) and a kind of physiological disintegration in the TCRS. In such a case, attention and vigilance are on the wane.

AA in hypnagogia is markedly differentiated with a conspicuous preponderance of high positivity shaped like hourglass in the central and frontal regions on the right. The picture is almost statically unchanging. This appears to be a paradoxical finding; the EEG curve is differentiated (as in "fractals") yet static at the same time (as in "attractors"). which, however is typical of hypnagogia (NR1).

C. $\mathbf{m} / \mathbf{n}=\mathbf{2} / \mathbf{1 9}$, representing a high degree of desynchronization in contrast to Fig 4, i.e. decreased vigilance due to "disintegration" in thalamocortical reverberation (TCR). 

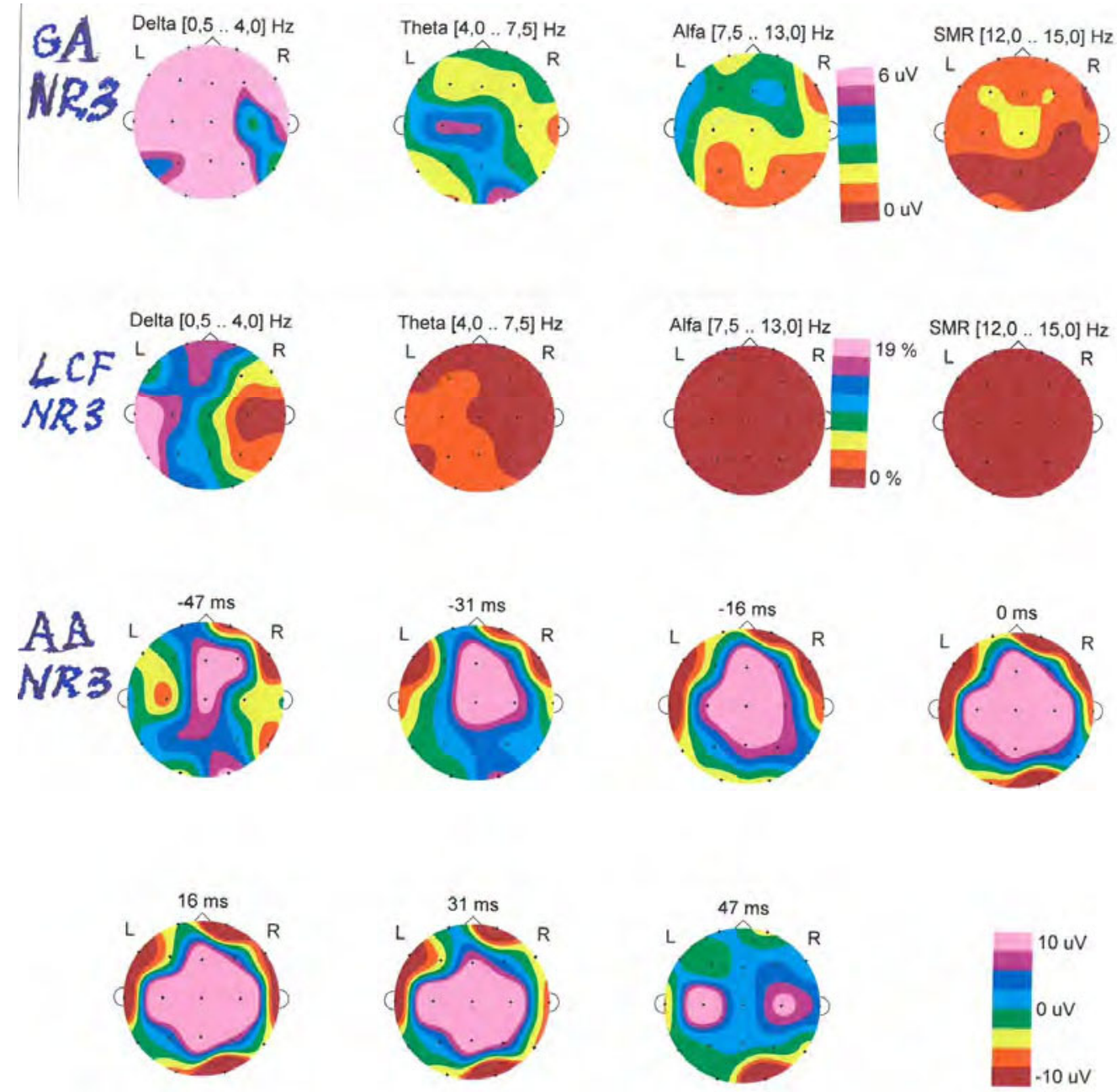

Figure 7. An analysis of the right side of EEG shown in Fig 5, or from deep sleep (NONREM 3) with delta waves.

The spectrum (GA) on the Figure 7 shows predominant diffuse delta aktivity as a sign of high synchronization of rhythm generators (positive wave interference) both in the thalamus and in the cortex. All that we find here is but a small focal theta over the left central region. Alpha shows, quite atypical, moderate frontal predominance which indicates very atypical localization of alpha, or rather alpha-like activity, perhaps resembling "mental kappa" rhythm. LCF in NONREM 3 is very asymmetrical with a predominance over the left hemisphere in the delta band. Apparently, not even in deep sleep do we meet the criteria of hemispheric symmetry but not so asymmetrically as dolphins or other cetaceans. Other frequences have, during LCF, similarly as during hypnagogia, nearly zero values. Obviously, physiological hypersynchronization (NONREM 3) is quite different from pathological (e.g. epileptic EP) hypersynchronization; also, the resemblance of an attractor is not so convincing in this case.

AA in NONREM 3 again shows cyclicity; at first, positivity is low, then dominant to decrease toward the 94th millisecond. The limits of this positivity are variable and not so constant as in epileptic paroxysm (EP). Nevertheless, EEG curve dedifferentiation in the direction of 
increased synchronization is obvious here. During NONREM 3, with regard to greater variability than in EP pictures, we analyzed the first 3 maps $(-47,-31,-16 \mathrm{~ms})$ and obtained the following results: c.m/n $=5 / 19$, in the first map, $6 / 19$ in the second map. and $8 / 19$ in the third map, and so on. In other words, synchonization increases prominently, which is why vigilance will be prominently suppressed. After all, in both states, i.e. deep sleep (NONREM 3 ) and paroxysm (EP) consciousness is considerabley altered, even though, in both states, the quality of the changes is substantially different: physiological in sleep and pathological during paroxysm.

Another example is the Figure 8 which shows two long horizontal lines in the EEG curve underline the basic alpha rhythm which is prominently more regular and higher over the right hemisphere. Asymmetry is a typical feature of ADHD. The short broken lines, often with superposed alpha activity, indicate episodes of delta also typical of this syndrome. Doubleline accentuation marks the site of AA analysis. The red line at the lower edge of the EEG indicates the interval of GA and LCF analysis.

The lower part of the picture carries GA maps with two foci of delta in the left temporal and occipital regions and brightly prominent asymetric alpha in the right temporooccipital area as corroborated by visual description of the EEG curve.

At the very bottom are maps of LCF analysis with slightly increased focal coherence in the right temporooccipital region with high coherence ibid. The finding is again confirmed by visual description of EEG.

The maps on the Figure 9 in the occipital middle region $(-16$ to $+16 \mathrm{~ms})$ show negativity with positivity shifted more temporalward. This is how alpha waves behave in the normal population $\mathrm{c} . \mathrm{m} / \mathrm{n}$ here is $\mathbf{4} / \mathbf{1 9}$.

The lower two rows of maps present an AA analysis of the alpha wave superimposed on the delta wave. No regular periodicity is discernible here, maximum negativity remains permanently in the temporal region and equally so permanent maximum positivity remains in the temporooccipital region on the right as evidence of certain rigid stability. Focal positivity is only in the vertex in central values in teh maps $(-16$ to $+16 \mathrm{~ms})$. C. $\mathrm{m} / \mathbf{n}$ moves between $2 / 19$ and $6 / 19$, suggesting lability of isovoltage and a tendency to hypersynchrony. We can see here contradiction between local adherence to maximum and minimum values and, on the other hand, their lability in intensity.

\section{DISCUSSION}

The $\mathrm{m} / \mathrm{n}$ coefficient in alpha activities is marked by rather lower values, in the paroxysm of absence in high values and in deep sleep in relatively (to absence) lower values. As we can see, this coefficient shows the state of synchronization, heterogeneity of EEG graphoelements and, we can say, also EEG curve integrity and, at the same time, the degree of vigilance. The ratio in the $\mathrm{m} / \mathrm{n}$ coefficient is an expression of not only periodicity, regularity and synchronization, but also of the rate of indeterminacy and, from the systemic point of view, the degree of freedom as well. Surprisingly, these values are not at their peak in full wakefulness; even during regular fusiform alpha activity, the coefficient is found at medium values. In contrast, the coefficients are high in states where consciousness is suppressed such as in deep sleep or paroxysm of absence. Attention disorders are marked in EEG by relatively typical changes at a rate of $90 \%$, mainly by episodes of delta waves following a relatively regular group of alpha waves, and tend to predominate over the posterior parts of the skull. Delta waves obviously mean interruption of alpha activity, which is why they are immediately connected with the psychic insufficiency of hyperactivity and non fluency of attention.

The cerebral system and graphic representation of its activity, i.e., trajectories are very intricate entities. EEG is a trajectory of the brain's electric field, containing pseudocyclicity and pseudostationarity; it has harmonic as well as random elements and properties of selflikeness. The results of spectral and coherence analyses suggest fractal properties of the EEG 
curve as most obvious in amplitude analysis of alpha activity. Hence, in certain states we can talk about trajectories of fractals, for example in wakefulness, and, in other situations, about attractors such as in epileptic paroxysms.

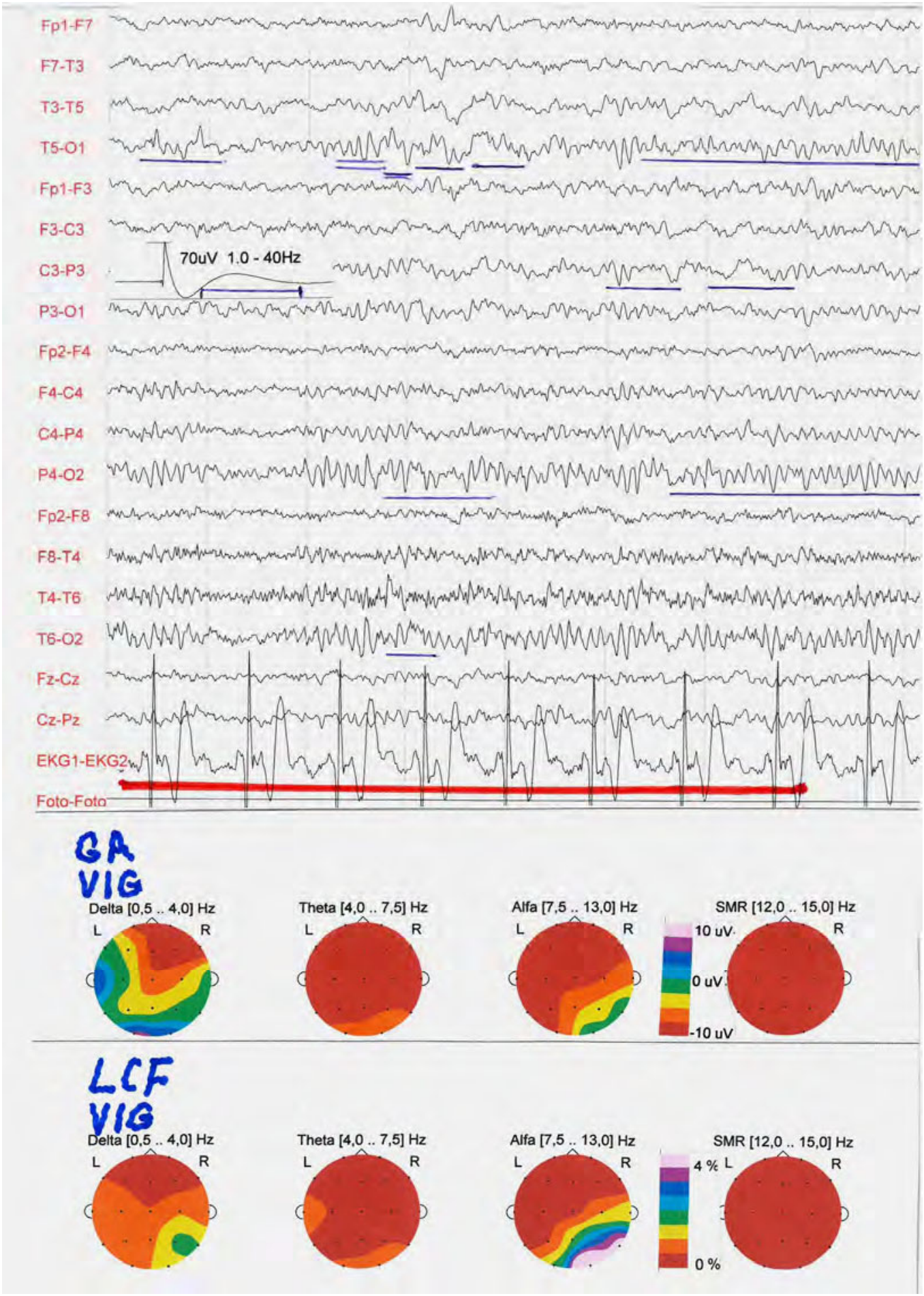

Figure 8. The upper part of the picture is 8-second long EEG of a 14-year-old boy with ADHD. 

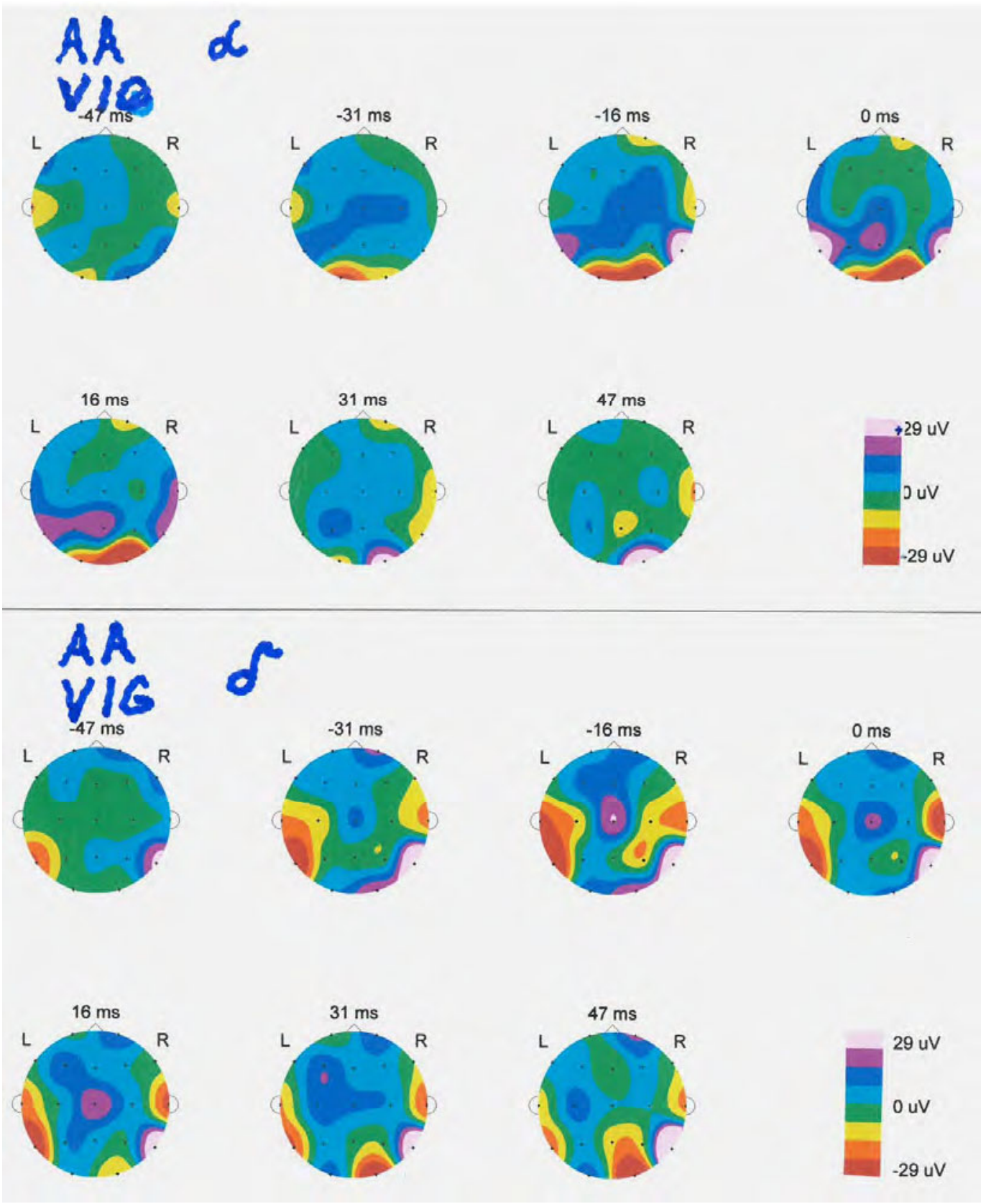

Figure 9. AA analysis of the same boy with ADHD shown in Figure 8. The upper two rows of maps show situations of isovoltage during a regular alpha wave (double-line accentuated site in the fourth EEG curve from the top in Fig.8. Discernible here is cyclicity the first map(-47ms) shows signs of positivity in the right temporooccipital region just as the last map (47ms) does.

The above method is designed to provide instructions for simplified identification of those psychological states. If we have a major quantity of data from a longer portion of the EEG curve, we shall apply further discrimination of data on the artificial neuronal network (ANN) 
which in themselves are good models for brain functions (BNN). Determination of trends in the current development of vigilance and EEG curve is important for the prediction of epileptic paroxysm or sleep activity. Moreover, there is mutual creative influencing and fertilization between ANN and BNN. Naud et al. (2008) modelled on ANN the genesis of epileptic spikes to find a relative simplicity of changes in the network during the genesis of epileptic graphoelements.

McCulloch and Pitts constructed in 1943 their perceptron based on their knowledge of the neuron; the device is in use to this day. It represents a rather simple mechanism with threshold and transmission functions, a number of inputs $\left(\mathrm{x}_{\mathrm{i}}\right)$ of different weights $\left(\mathrm{w}_{\mathrm{i}}\right)$ and a single output. ANN serve well as diagnostic means with adequate predictive power of trends in meterology, banking and also in medical diagnosis.

Using a smaller or larger number of perceptrons we can organize single-layer or multilayer networks. In essence, ANN operate so that they learn to know characters designed to identify networks or problems such as they are expected to solve. Just as in human learning, repetition is essential. For example, in one episode a picture has to be stored in memory, in the next episode it must be compared with the real, ideal picture. As a rule the two pictures differ from one another, which is why the memorized picture has to be specified, and this is done by repetition, iteration of comparation, mainly in TCRS (thalamo-cortical reverberation system) and hippocampus. This approximation proceeds until the two pictures become nearly identical. This means reaching anything up to $95-99 \%$ of precision and our BNN (from energy function point of view) are then in "global minimum". Having reached a lower degree of precision, e.g. only $60-80 \%$,, we can describe it as "local minimum" and our knowledge (analysis, cognition) is then but very unreliable.

In human memory and mind, the pictures are usually pseudoidentical with external "ideal" pictures, merely resembling them, mainly in areas of only marginal interest to us. For instance, as engineers, craftsmen, physicians, masons or painters we take only occasional interest in classic French or German literature. These are in our memory and knowledge in "local minimum", vague, fuzzy. However, in our own field we keep our knowledge well classified, we have a good idea of it, its pictures in our memory are good, as for the organization of our knowledge they are within the "global minimum". Such processes and their results came to being by multiple repetition in the TCRS. They are stored in our memory as a fixed but dynamic attractor. If we fail to use it, it will burn out as any other conditioned reflex. Attractors of this kind include not only simple conditioned reflex or dynamic stereotype but also the very complex Ukhtomsky dominant or strong, nearly obsessive emotively motivated behaviour, e.g. falling in love, creative passion for some problem, etc.)

In ANN we use as help what is known as energy function - Ef (Snorek, 2004).

Ef estimates the degree of informedness of the net, which means that it sets the difference between the real value of engram fixation in the network memory after the course of the training set and the required (ideal) value at the neuronal network outputs. Instead of Ef we can also use the term network error. As the network learns, the network error or energy diminishes. In ANN, the rate of informedness comes with changes in the thresholds and weights at the network inputs. Hence the energy function is as follows: $(\rightarrow=$ vector)

$$
\mathrm{E}=\mathrm{g} \underset{(\mathrm{W}, \vec{\Theta}, \vec{X}),}{\rightarrow}
$$

where $\mathrm{W}$ are vectors for the size of weights, $\Theta$ vectors for the size of thresholds,

and $\vec{X}=$ constant value of the ideal model. For better understanding of these reflections. Snorek (2004) presents a comparison with our walk in a hilly countryside. The above mentioned energy function can be imagined as a curved surface (bumpy countryside with a mountain range) in hyperspace. The current values of weights and thresholds are coordinates on this surface with energy being a point on the surface. We try to reach the point with a minimum of energy. It resembles a tourist hike in the mountains at the least visibility.We 
intend to reach our destination in a valley, i.e. along the greatest gradient. Consequently these functions belong among gradient methods. In neurophysiology this comparison tallies with memory function with threshold lowering $\Theta$ for easier recollection of certain stimuli under certain conditions such as, e.g., post-tetanic potentiation or kindling or simply recollection from memory. Regrettably, the currently available EEG methods do not allow of the above functions such as fractals, attractors or energy function use also for clinical or educational psychophysiology,

In connection with the brain's electrical activity and its EEG trajectory, let us bring to mind some facts about the characteristicts of "unpredictable" systems. Fractals and attractors are terms from the new science of chaodynamics, i,e, about the behaviour of complex systems which are not simply predictable, which contain mostly harmonic as well as random (noise) components, which are present simultaneously or successively or alternating intermittently (Gleick 1995; Šíma \& Neruda, 1996; Novák et al.,1998; Šnorek, 2004; Voráčová et al.,2013). Systems of this kind include, e.g. our stellar galaxy, climate on our Earth or also our brain.

Fractals have different definitions, e.g. geometrical formation (curve, set of points) whose topological dimension is a smaller Hausdorff dimension. To give an example, the circle is a classic topological curve, all of its points lying at the same distance from the centre. In terms of Hausdorff's dimension, the circle is formed by iterative rotation of the triangle (by repeating (iterating) a similar mode of calculation). The Koch snowflake results after a small number of triangle rotations, though after a large number of iterations it is hard to differentiate from a smooth-surfaced circle, though in fact the circle circumference consists of ever smaller tiny triangles invisible to the naked eye.

Parts of fractals are like the whole and themselves, exhibiting "self-likeness", usually independent of the scale.

According to the Kolmogorov - Mandelbrot formula $\left(D_{\text {КM }}=\log N / \log k\right)$ The Koch curve has a fractal dimension equal to 1,2619. (If in each iteration the picture (abscissa) is zoomed to one third (k) the picture will consist of $4(\mathrm{~N})$ smaller pictures.) Oddly, unlike classic dimensions, these are non-whole-number dimensions. Only the famed Mandelbrot set has a dimension ( $\left.D_{\text {Км}}\right)$ equal to 2 . A modified Koch curve arisen in irregular alternation of the sides may resemble a complex EEG curve. A justifiable question for electroencephalography is as follows: would the $D_{\mathrm{KM}}$ curve estimation make sense where our analyses so far in comparison with the clinical picture have failed? It is our cherished hope that in the future we shall be able to determine this dimension or energy function of the EEG curve, and that we shall be able to make objective assessments of the state of informedness of the brain's neuronal population.

All attractors are fractals, but not all fractals are attractors. Attractors can be viewed as patterns resulting from regular contraction with the aid of iterative function system (IFS). Attractors showing the behaviour of simple as well as highly complex systems such as when the pendulum stops swinging, its trajectory becomes a point in what is a point attractor. If the pendulum has a steady supply of energy, it keeps swinging incessantly in what is a cyclic attractor. This applies also to complex systems such as the revolutions of a number of celestial bodies around a common centre of gravity, daily or annual fluctuation of temperature or biochemical processes. As Prof. Laufberger once said (1947), life is a set of fixed cyclic enzymatic reactions. Cellular metabolism is a process so intricate that its trajectory would be very complex; hence we describe it as a strange (mirabilis) attractor. The EEG curve is also quasistationary and quasiperiodic, which why it escapes our "linear" analysis. The complexity of the EEG curve is determined by biochemical, metabolic, psychic and social effects on the metamorphoses of the brain's activity. That accounts for the occasional attractor behaviour, e.g. epileptic graphoelements of spike-wave or deep sleep NONREM 3, at other times fractal behaviour, e.g. contemplation, NONREM 1 or REM sleep. 


\section{ACKNOWLEDGEMENT}

The author thanks for the support by the project PRVOUK and SVV provided by Charles University.

\section{REFERENCES}

Ambler, Z., Bednař́k , J., \& Růžička E. et al. (2004). Klinická neurologie (Clinical Neurology). Triton, Praha. (in Czech).

Atwood, H. L. \& MacKay A. (1989). Essentials of Neurophysiology. Toronto, Philadelphia, Decker Inc.

Bob, P. (2005). Psychobiology of dissociation and relationship between epilepsy and mental illness. Acta Universitatis Carolinae Medica, Monographia. CLI, Charles University in Prague.

Cragg, B.,G. (1975). The Development of Synapses in the Visual System of the Cat. Journal of Comparative Neurophysiology,160, 147-166.

Faber, J. (1978). Vigilance, sleep, petit mal and EEG as manifestation of programmed brain regulation. 1st ed. Praha: Acta Universitatis Carolinae Medica, Monographia LXXXVII.

Faber, J., \& Vladyka, V. (1984). Nocturnal sleep stereo-electroencephalography and polygraphy in epileptics. 1st ed. Praha: Acta Universitatis Carolinae Medica, Monographia, CVIII.

Faber, J. (2005) QEEG. 1st ed. Praha, Galén.

Faber, J. (2015). Bulbo-thalamo-cortical structures and their activities simulated by "formator-complex" and "multilayered iterative algorithm" models. Praha, Acta Universitatis Carolinae Medica, Monographia CLX.

Gleick, J. (1995). Chaos. 1st ed. Brno, Ando Publishing.

Kukleta M., Lamarche M., \& Louvel J. (2009). Component N400 Can Be Demonstrated In The EventRelated Potential From a Simple Auditory Reaction Time Paradigm. Activitas Nervosa Superior, 51, 1-5.

Laufberger V. (1947). Vzruchová teorie. (Impuls Theory.) 1st ed. Praha, Nákladem spolku českých lékařro. (in Czech).

Lion, K.S., \& Winter, D.F. (1953). A method for the discrimination between signal and random noise of electrobiological potentials. Electroencephalography and clinical Neurophysiolology, 5, 109-113. Naud, R., Marcille, N., Clopath C., \& Gerstner, W. (2008). Firing patterns in the adaptive exponential integrate-and-fire model. Biological Cybernetics, 99, 335-347.

North, G., \& Greenspan, R.J. (2007). Invertebrate Neurobiology. 1st ed. New York: Cold Spring Harbor Laboratory Press.

Novák M, et al. (1998). Arteficial Neuronal Networks. 1st ed. Praha: CH Beck. (in Czech).

Petrovický, P., Brabec, J., \& Faber, J. et al. (2008). Clinical Neuroanatomy. Triton, Praha.

Rimbaud, L. (1957). Préci de Neurologie, G. Doin et Cie, Paris.

Saunders, M.,G. (1963). Amplitude probability density on alpha-like patterns. Electroencephalography and clinical Neurophysiology, 15, 761-765.

Šíma, J, \& Neruda, R. (1996). Teoretické otázky neuronových sítí. 1st ed. Praha: Matematicko - fyzikální fakulta UK Praha. (in Czech).

Šnorek, M. (2004). Neuronal networks and neurocomputers. 1st ed. Praha: FEL ČVUT.

Trojan, S, \& Št'astný, F. (1980). Ontogenesis of the brain. Vol. 3, Praha: Universitas Carolina Pragensis.

Valverde, F. (1971). Rate and extent for recovery dark rearing in the visual cortex of the mause. Brain Reasearch, 33, 1 - 11.

Vitek, J. (1951). General Neurology. 1st ed. Praha, SZN.

Voet, D, \& Voetová, J. (1900). Biochemistry, 1st ed. Praha: Victoria Publishing (in Czech).

Voráčová, Š, et al. (2013). Atlas of geometry. 1st ed. Praha: Academia. (in Czech). 